\title{
Adrenergic Modulation With Photochromic Ligands
}

\author{
Davia Prischich, Alexandre M. J. Gomila, Santiago Milla-Navarro, Gemma Sangüesa, \\ Rebeca Diez-Alarcia, Beatrice Preda, Carlo Matera, Montserrat Batlle, Laura Ramírez, \\ Ernest Giralt, Jordi Hernando, Eduard Guasch, J. Javier Meana, Pedro de la Villa, and \\ Pau Gorostiza*
}

\begin{abstract}
Adrenoceptors are ubiquitous and mediate important autonomic functions as well as modulating arousal, cognition, and pain on a central level. Understanding these physiological processes and their underlying neural circuits requires manipulating adrenergic neurotransmission with high spatio-temporal precision. Here we present a first generation of photochromic ligands (adrenoswitches) obtained via azologization of a class of cyclic amidines related to the known ligand clonidine. Their pharmacology, photochromism, bioavailability, and lack of toxicity allow for broad biological applications, as demonstrated by controlling locomotion in zebrafish and pupillary responses in mice.
\end{abstract}

\section{Introduction}

Adrenergic neurotransmission plays an essential role over the unconscious regulation of most vital functions in the human body. Heart and respiratory rate, digestion, smoothmuscle contraction, gland secretion, and pupil diameter are modulated by noradrenaline release from sympathetic fibres projecting from the peripheral nervous system (PNS). ${ }^{[1]}$ The neurotransmitter role is not limited to the PNS, though. Adrenergic neurons firing from the locus coeruleus (LC) towards different areas of the central nervous system mediate alertness, responses to acute stress and danger, pain modulation, arousal, sleep-wake cycles, as well as neuroplasticity and cognitive behaviour. ${ }^{[2-4]}$ Despite the physiological relevance of adrenergic neurotransmission, tools to precisely modulate its activity and to functionally dissect adrenergic pathways have been scarce. Classical pharmacology, which benefits from high receptor specificity, is inadequate to address cellular processes with high spatio-temporal selectivity. In this regard, light offers a non-invasive and versatile mean to precisely control a variety of biological targets. Photocontrol of adrenergic signalling has been shown with optogenetics $^{[5-7]}$ and photo-uncaging. ${ }^{[8]}$ However, while the first technique requires genetic manipulation and can lead to unintended neuroplastic phenomena, the irreversible release of caged ligands displays diffusion-limited resolution and is challenging in vivo. Photopharmacology poses an alternative to precisely and reversibly deliver drug activity in space and time by introducing photochromic groups into the structure of bioactive compounds. ${ }^{[9,10]}$ Successful examples of photoswitchable drugs include ligands of ion channels, receptors and enzymes, peptides, lipids and nucleic acids. ${ }^{[11-13]}$ However, compounds to photoswitch adrenergic neurotransmission are
[*] D. Prischich, A. M. J. Gomila, B. Preda, C. Matera, P. Gorostiza Institute for Bioengineering of Catalonia (IBEC), The Barcelona Institute for Science and Technology (BIST)

Barcelona (Spain)

D. Prischich, A. M. J. Gomila, C. Matera, P. Gorostiza

Centro de Investigación Biomédica en Red-Bioingeniería, Biomateriales y Nanomedicina (CIBER-BBN) (Spain)

S. Milla-Navarro, L. Ramírez, P. de la Villa

Department of Systems Biology, University of Alcalá (UAH)

Madrid (Spain)

G. Sangüesa, M. Batlle, E. Guasch

Institut Clínic Cardiovascular, Hospital Clinic, University of Barcelona (UB), Institut d'Investigacions Biomèdiques August Pi i Sunyer (IDIBAPS)

Barcelona (Spain)

and

Centro de Investigación Biomédica en Red—Enfermedades Cardiovasculares (CIBER-CV) (Spain)

R. Diez-Alarcia, J. J. Meana

Department of Pharmacology, University of the Basque Country (UPV/EHU)

Leioa, Bizkaia (Spain)

and

Centro de Investigación Biomédica en Red-Salud Mental (CIBER-

SAM) (Spain)

\author{
E. Giralt \\ Department of Inorganic and Organic Chemistry, University of \\ Barcelona (UB) \\ Barcelona (Spain) \\ and \\ Institute for Research in Biomedicine (IRB), Barcelona Institute for \\ Science and Technology (BIST) \\ Barcelona (Spain) \\ J. Hernando \\ Departament de Química, Universitat Autònoma de Barcelona \\ (UAB) \\ Cerdanyola del Vallès (Spain) \\ P. de la Villa \\ Instituto Ramón y Cajal de Investigación Sanitaria (IRYCIS) \\ Madrid (Spain) \\ P. Gorostiza \\ Catalan Institution for Research and Advanced Studies (ICREA) \\ Barcelona (Spain) \\ E-mail: pau@icrea.cat \\ (46) Supporting information and the ORCID identification number(s) for \\ the author(s) of this article can be found under: \\ https://doi.org/10.1002/anie.202010553.
}

(iD) 
still not available. In this study we aimed at developing adrenergic drug-like molecules that can be reversibly controlled with light.

\section{Results and Discussion}

Among the large database of sympatholytic and sympathomimetic agents, we focused on a class of cyclic amidines structurally related to clonidine and other commercialized drugs targeting $\alpha$-ARs (Figure 1A). All adrenergic agents share a common pharmacophoric motif: a primary or secondary aliphatic amine, protonated at physiological $\mathrm{pH}$,

A

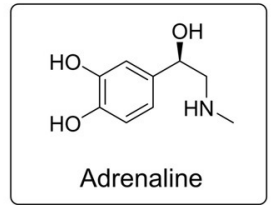<smiles>Clc1cccc(Cl)c1NC1=NCCN1</smiles><smiles>Clc1cccc(Cl)c1C(Cl)C1NCCN1</smiles>

Lofexidine<smiles></smiles>

Cirazoline<smiles></smiles>

Medetomidine

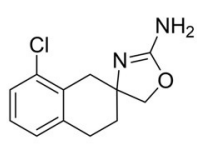

S18616

B
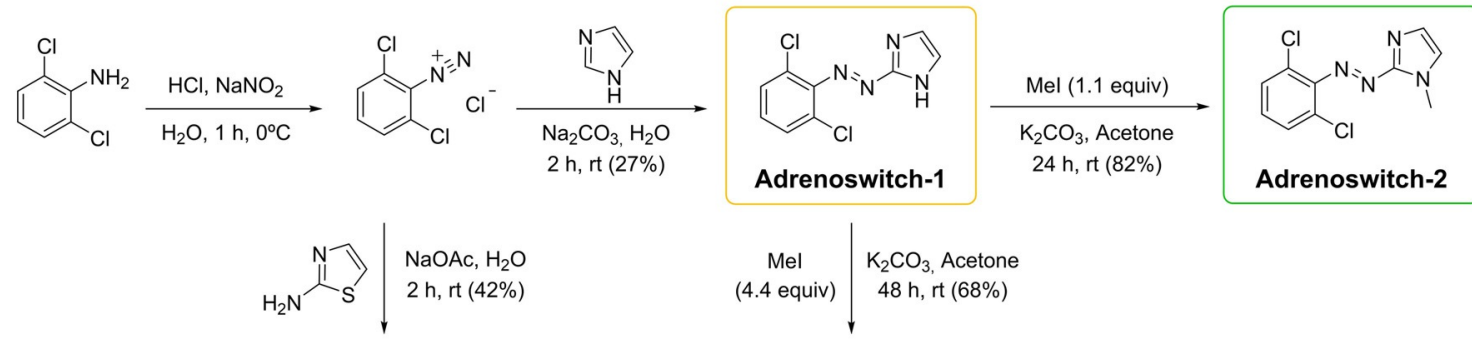

$$
\mathrm{Cl}_{\mathrm{Cl}}^{\mathrm{Cl}} \mathrm{N}_{\mathrm{S}}^{\mathrm{N}}-\mathrm{NH}_{2}
$$

Adrenoswitch-4<smiles>Cn1cc[n+](C)c1/N=N/c1c(Cl)cccc1OCCO</smiles>

C
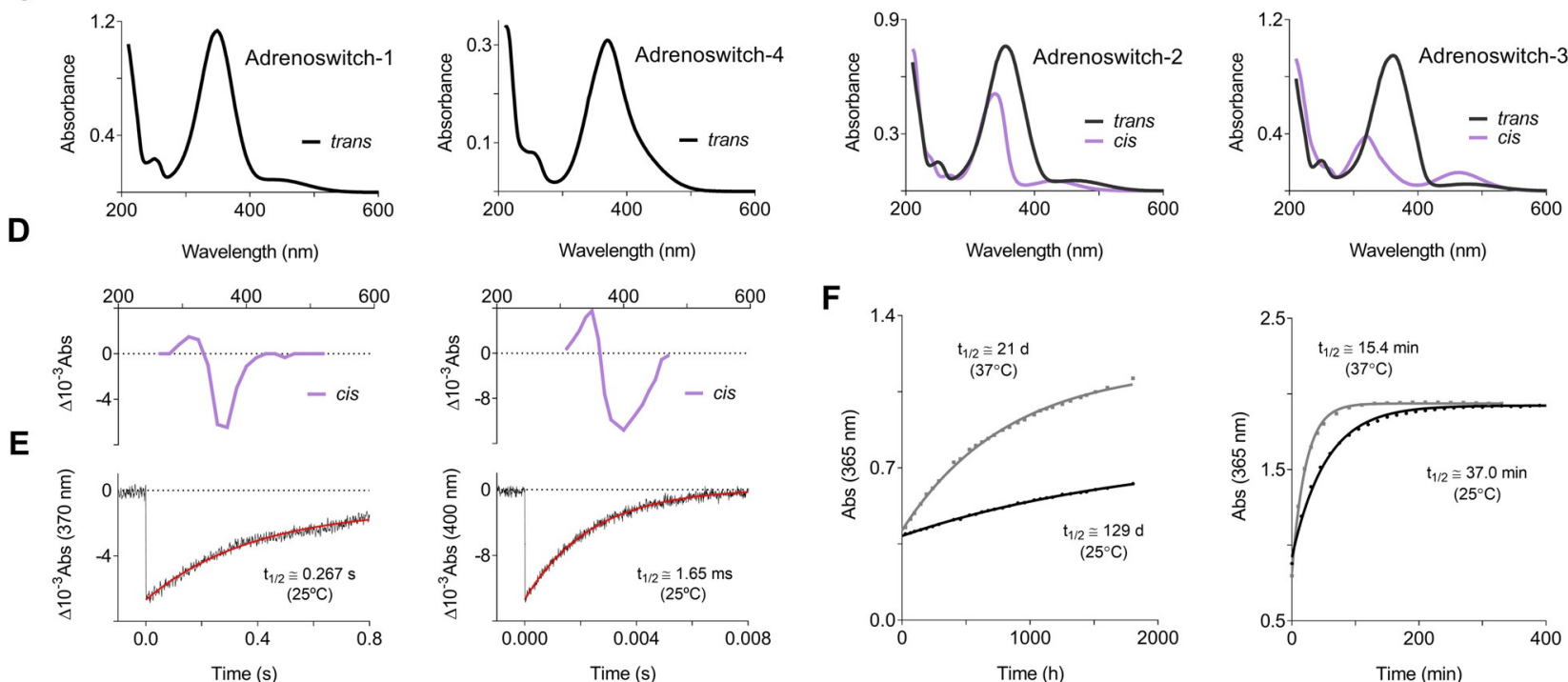

600

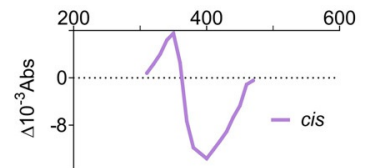

$\mathbf{F}$
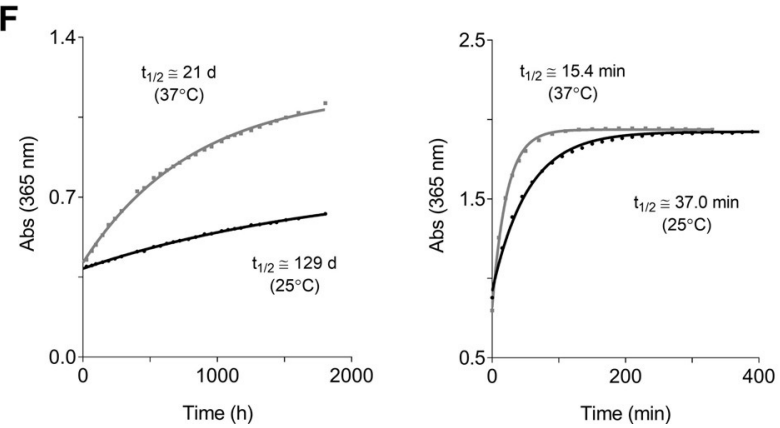

Figure 1. A) Chemical structures of adrenaline and some of the synthetic adrenergic ligands that inspired this work. B) Divergent chemical synthesis of adrenoswitches 1-4. C) UV/Vis absorption spectra of the trans isomers. For slow-relaxing adrenoswitch-2 and -3, UV/Vis absorption spectra of the cis isomers are also reported. Spectra were extracted from UPLC chromatograms after elution with a mixture of water and acetonitrile supplemented with trifluoroacetic acid. Spectra of cis and trans isomers were normalized at their isosbestic points (see SIFigures S3A-C). D) Transient absorption spectra of adrenoswitch-1 and -4 upon pulsed excitation at $\lambda_{\text {exc }}=355 \mathrm{~nm}$ in physiological buffer ( $\mathrm{pH} 7.4$, $25^{\circ} \mathrm{C}$ ). E) Absorption loss and recovery kinetics of adrenoswitch-1 and adrenoswitch-4, measured at $\lambda=370$ and $400 \mathrm{~nm}$, respectively, upon irradiation with a single ns laser pulse $(t=0)$ at $\lambda_{\text {exc }}=355 \mathrm{~nm}$ in physiological buffer $\left(\mathrm{pH} 7.4,25^{\circ} \mathrm{C}\right)$. Red lines correspond to monoexponential fitting of the experimental data. F) Cis-to-trans thermal relaxation of adrenoswitch-2 and -3 at $25^{\circ} \mathrm{C}$ (in black) and $37^{\circ} \mathrm{C}$ (in gray) under dark conditions. Data were fitted to a monoexponential decay model for cis isomers half-lives determination. 
and separated by a short linker from a substituted benzene ring. ${ }^{[14]}$ We postulated that $\alpha$-adrenergic ligands were suitable candidates for "azologization" ${ }^{[15,16]}$ as the structural changes required to obtain photo-responsive derivatives could be introduced without altering essential pharmacophoric elements. In addition, the prospect of obtaining dark-inactive adrenergic azologs was supported by the lack of biological activity of trans-like epoxydic analogues compared to cis-like compounds as described in the work of Gentili et al. ${ }^{[17]}$ We thus designed a set of putative photoswitchable adrenergic ligands, named "adrenoswitches", by replacing the short linker with an azo group while constraining the cyclic amidine moiety to the closest structurally related aromatic derivatives. The ortho-dichlorobenzene system, common to clonidine and lofexidine, was maintained to favour both adrenergic activity and photochromism. First, halogen substitution increases the lipophilicity of a molecule, thus improving its absorption and its permeability to the blood brain barrier or the blood ocular barrier. ${ }^{[18]}$ Moreover, lipophilic substituents at the positions 2 and 6 of the phenyl ring are well tolerated in terms of pharmacological activity by both $\alpha_{1}$ - and $\alpha_{2}$-ARs. ${ }^{[19]}$ Secondly, the photochromism of ortho-halogenated azobenzenes offers enhanced thermal stability, longer photoisomerization wavelengths, and higher isomerization ratios. ${ }^{[20,21]}$

To identify suitable aromatic heterocycles, in our first analogue (adrenoswitch-1, Figure 1B) we substituted the imidazoline ring with an imidazole. As phenylazoimidazoles are known to undergo fast cis-trans thermal isomerization (i.e. fast-relaxing photoswitches), in adrenoswitch-2 we sought to reduce the rate of this process with an $N$-methyl imidazole. ${ }^{[22]}$ In adrenoswitch-3 we introduced a $N, N$-dimethyl imidazolium, as a permanent positive charge might better mimic the electronic properties of the original cyclic amidine in its physiologically protonated form. A similar strategy for the same purpose was using 2-aminothiazole in adrenoswitch-4.

Our library was prepared via a divergent synthetic approach involving a standard azo coupling reaction (Figure 1B). Commercially available 2,6-dichloroaniline was converted into the corresponding diazonium salt and reacted under mild basic conditions either with imidazole to provide adrenoswitch-1 or with 2-aminothiazole to afford adrenoswitch-4. Adrenoswitch-2 and adrenoswitch-3 were obtained from adrenoswitch-1 through reactions of mono- and di- $N$ methylation, respectively.

As these photoswitches have not been described among the reported azoheteroarenes, ${ }^{[23]}$ their photochromic behaviours were then investigated. Slow-relaxing adrenoswitch-2 and -3 were characterized by steady-state UV/Vis absorption spectroscopy (Figures 1 C,F), while transient UV/Vis absorption spectroscopy was used for fast-relaxing adrenoswitch1 and -4 (Figures 1D,E). Our compounds can be photoisomerized from trans to cis with (ultra)violet light (365$400 \mathrm{~nm}$ ) and from cis to trans with blue or green light (450 $500 \mathrm{~nm}$ ). As intended by design, thermal relaxation rates vary considerably along the series. Measured half-lives spanned from milliseconds (adrenoswitch-4) to seconds (adrenoswitch-1), minutes (adrenoswitch-3), and months (adrenoswitch-2) (Figures 1E,F).
With all the information to effectively photoswitch our ligands, we assessed their biological activity as a function of illumination. The affinity of the library towards $\alpha_{2}$-ARs was measured by competitive radioligand binding assay in prefrontal cortex membranes obtained post mortem from human brains. All the adrenoswitches competed in binding to the receptor albeit at weaker affinities than clonidine. Most notably, slow-relaxing adrenoswitch-2 and -3 showed a significant change in binding potency upon UV illumination (Figure $2 \mathrm{~A}$ and Figures S4 A,B). On the other hand, no difference in binding could be observed for adrenoswitch1 and -4 , possibly because these compounds display fast thermal isomerization and the binding assay might reflect mainly the properties of the trans isomers. Nonetheless, since in the work of Gentili et al. trans and cis-like epoxy analogues showed similar binding affinities, but produced different biological activities,${ }^{[17]}$ we decided to further test both slowand fast-relaxing adrenoswitches.

Since the adrenergic system is a major regulator of the vascular tone, we characterized the adrenergic effects of our compounds by measuring their vascular activity in ex vivo rat aortic rings. ${ }^{[24-26]}$ Besides being generally recognized as a potent central hypotensive agent, ${ }^{[27]}$ clonidine is also known to induce vasodilation by acting directly on vascular adrenoceptors. ${ }^{[25]}$ This peripheral response involves stimulation of endothelial $\alpha_{2}$-ARs as well as partial agonism towards $\alpha_{1}$-ARs of the vascular smooth muscle, and results in relaxation of endothelium-intact strips previously contracted with phenylephrine (PE). ${ }^{[26,28-30]}$ When testing if our analogues displayed a similar behaviour, we observed that exposure to UV light alone could induce relaxation of the vascular smooth muscle, substantially abolishing the contraction evoked by $\mathrm{PE}^{[31]}$ These intrinsic photoresponses are due to nitric oxide (NO) release either from endogenous or exogenous donors or from storage vesicles. $^{[32,33]}$ To minimize unintended photorelaxation, we pre-incubated the vessels in a suitable nitric oxide synthase (NOS) inhibitor, namely $N^{\mathrm{G}}$-methyl-L-arginine acetate (L-NMMA) which is reported to be unaffected by light. ${ }^{[34]}$ Remarkably, all adrenoswitches displayed high vasodilatory efficacies (maximal evoked response $E_{\max }$, Figure 2B) with adrenoswitch-1 coming through as the most potent compound and the only one displaying a lightdependent behaviour in this assay $(3 \times$ increase in potency upon UV illumination, Figure S8A). Although this assay was useful to identify and rank the adrenergic efficacy and potency of adrenoswitches, it had important shortcomings. Intrinsic photoresponses forced us to use a NOS inhibitor, thus partially blocking endothelium-dependent relaxation responses to $\alpha_{2}$-AR agonists. ${ }^{[26,35]}$ Moreover, the model proved complex to clarify adrenoswitches vascular pharmacodynamics as the observed antihypertensive responses might involve both $\alpha_{1}$ and $\alpha_{2}$-ARs and do not exclude further contributions from other non-adrenergic targets, most notably imidazoline receptors. ${ }^{[36-38]}$

We next characterized the activity of adrenoswitches in vivo. As proof-of-concept applications, we used the most promising compound of the series, adrenoswitch-1, to remotely manipulate locomotion in zebrafish and pupillary responses in mice. 
A

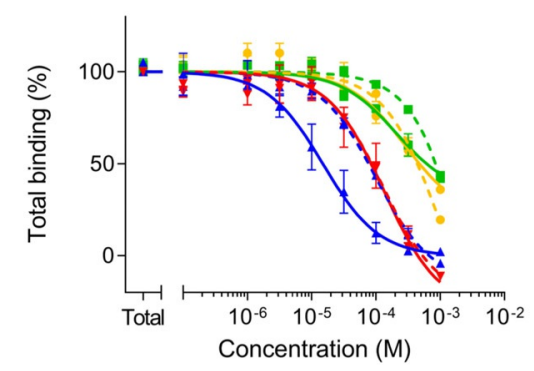

C

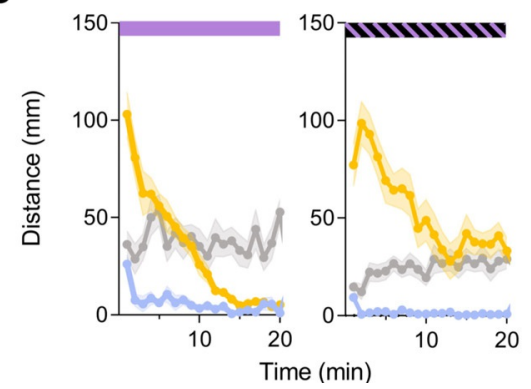

B

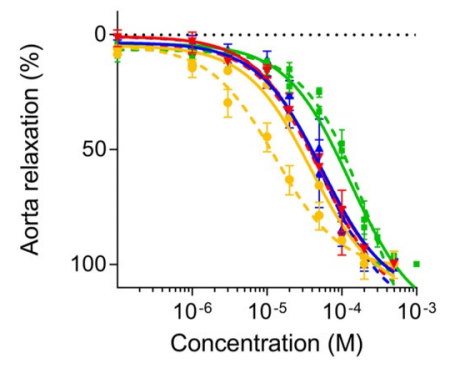

D

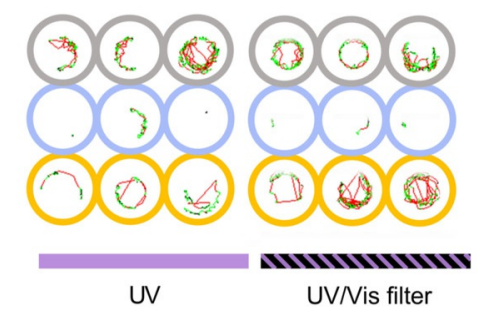

Legend

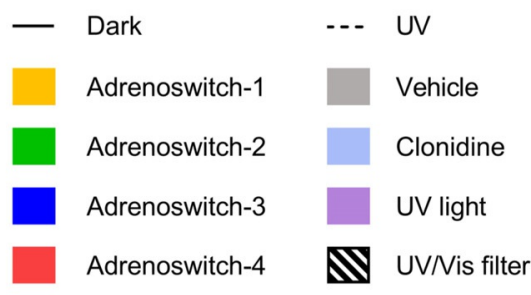

E

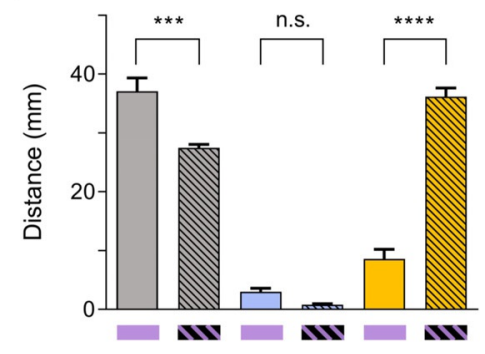

Figure 2. A) Competition binding curves of dark-relaxed (trans, solid lines) and UV-illuminated (cis, dashed lines) adrenergic photoswitches to $\alpha_{2}$ ARs in human brain pre-frontal cortex tissue. Binding is expressed as percentage of radioligand- $\left.{ }^{3} \mathrm{H}\right] \mathrm{RX} 821002-\mathrm{bound}$ to the receptor in the presence of the competitor. Data are means \pm SEM of two independent experiments run in duplicates or triplicates. B) Dose-response curves comparing the vasodilatory potencies of dark-adapted (trans, solid lines) and cis-enriched (dashed lines) adrenoswitches administered in the dark or under UV illumination to rat aortic rings where the endothelium had been preserved. Vessels were pre-contracted with $10^{-6} \mathrm{M}$ phenylephrine (PE) after treatment with $10^{-3} \mathrm{M} \mathrm{N}^{\mathrm{C}}$-methyl-L-arginine acetate (L-NMMA), a nitric oxide synthase inhibitor. Relaxation is expressed as percentages of the reference contraction induced by PE. Data are means \pm SEM (adrenoswitch-1, $n=4$ (per condition); adrenoswitch-2, (trans) $n=6$ (cis) $n=5$; adrenoswitch-3, (trans) $n=3$ (cis) $n=4$; adrenoswitch-4, $n=2$ (per condition). C,D) Danio rerio 7 days post-fertilisation larvae swimming distances and trajectories (movements with velocities over $6 \mathrm{~mm} \mathrm{~s}^{-1}$ ) after treatment with vehicle (grey wells), $50 \mu \mathrm{M}$ clonidine (light-blue wells) and $50 \mu \mathrm{M}$ adrenoswitch-1 (yellow wells). Conditions were simultaneously analysed under $365 \mathrm{~nm}$ UV illumination (left panels, cis-enriched adrenoswitch-1) and under a UV/Vis filter (right panels, trans adrenoswitch-1) that only transmits infrared light for movement recording. E) Quantification of the swimming distances shown in (C) at $t=20$ minutes. Data are means \pm SEM ( $n=12$ per treatment). Statistical differences between UV- and non-UV-exposed larvae were determined by two-way ANOVA with Tukey's multiple comparison test (n.s., not significant; **'*, $p$ value $<0.01 ; * * * *, p$-value $<0.001)$.

Zebrafish (Danio rerio) is a well-established animal model for both neurobehavioural ${ }^{[39]}$ and toxicological studies, ${ }^{[40]}$ and the transparency of their larvae makes them convenient for photopharmacological applications. ${ }^{[41,42]} \mathrm{Be}-$ sides, the expression and function of zebrafish ARs have been studied ${ }^{[43]}$ and the effect of dexmedetomidine, a central-acting and structurally-related $\alpha_{2}$-AR agonist, has been described by Ruuskanen et al. ${ }^{[44]}$ In analogy to the sedative effect reported for dexmedetomidine, clonidine $(50 \mu \mathrm{M})$ caused locomotor inhibition when compared to untreated animals (Figure 2CE). Clonidine effect was not significantly altered by UV light, although in vehicle-treated animals we observed an increase of the swimming distances as a result of their photomotor response to illumination. ${ }^{[39]}$ In contrast, larvae treated with adrenoswitch-1 $(50 \mu \mathrm{M})$ responded to UV light by progressively reducing their locomotion to levels comparable to clonidine-treated animals under UV (Figures 2C-E and Figures S9,S10). Therefore, adrenoswitch-1 displayed a clonidine-like behaviour upon UV-activation, while its trans isomer was unable to evoke sedation. Although this response is unequivocally light-induced, thermal relaxation of the compound was not enough to revert the behavioural effect (Figure 1E and Figure S10). Since other non-adrenergic receptors might evoke locomotion responses, we further studied adrenoswitch-1 in another in vivo model.

Among the available animal models for assessing autonomic responses, we sought one in which adrenoceptors played a major physiological role and in which the use of photoswitchable drugs may have actual investigational and therapeutic implications. Modulating pupil responses fulfilled both criteria and it seemed particularly attractive as the target tissue is naturally accessible to light. In addition, pupillometry allows for a selective evaluation of $\alpha$-adrenergic activity and it excludes the involvement of imidazoline receptors. ${ }^{[45-47]}$ As pupils adapt their diameter to changes in light intensity (pupillary light reflex, PLR), we resorted to genetically engineered blind mice (Opn4xRd10) for a first assessment of the compound activity. Opn $4 x R d 10$ mice do not express melanopsin and their rods and cones degenerate two months after their birth, thus becoming physiologically insensitive to any luminous stimuli. We tested if adrenoswitch- 1 enabled photoregulation of pupil diameter after topical administration $(1 \mathrm{mM}, 0.02 \% \mathrm{w} / \mathrm{v})$ to isoflurane-anesthetized animals. The compound exerted mydriasis only under UV illumination and the effect fully reversed in the dark, when adrenoswitch1 rapidly relaxes to the trans configuration (Figures $3 \mathrm{~A}-\mathrm{C}$ 
A
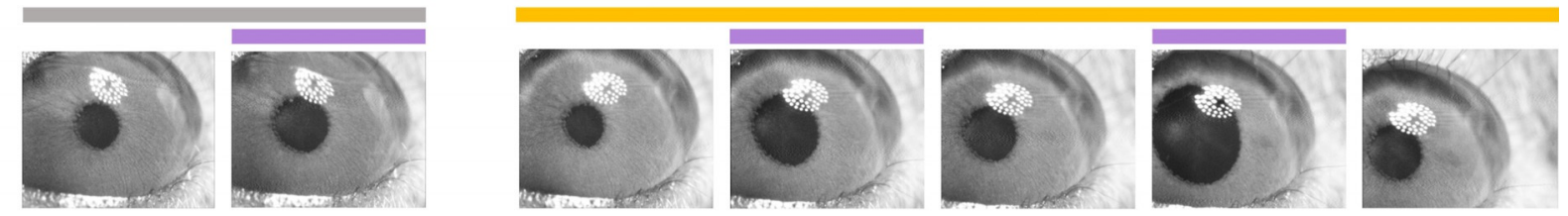

B

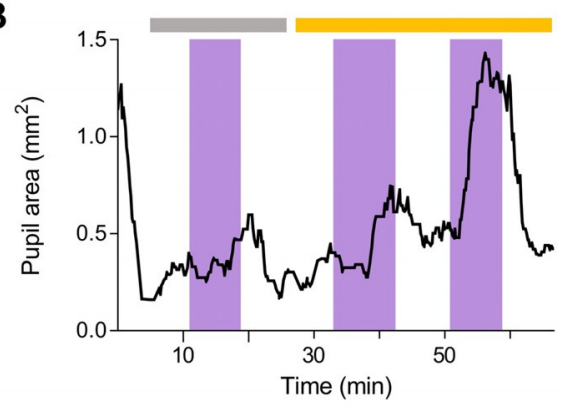

D

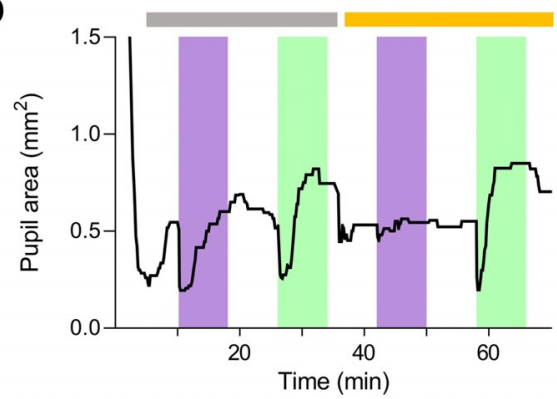

C

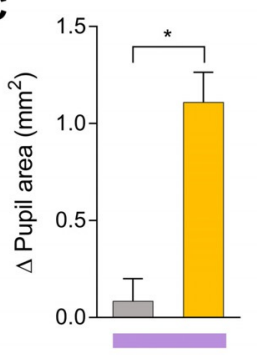

E

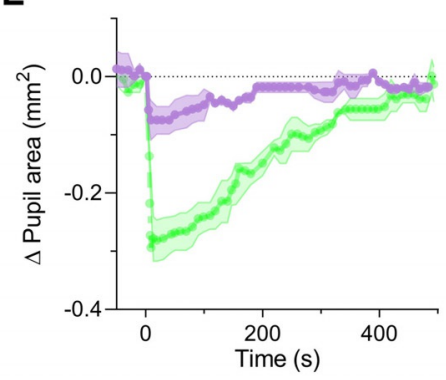

Legend

Adrenoswitch-1 UV light

Vehicle $\quad$ Visible light

Clonidine $\quad \mathbf{N}$ UV-Vis filter

$\mathbf{F}$

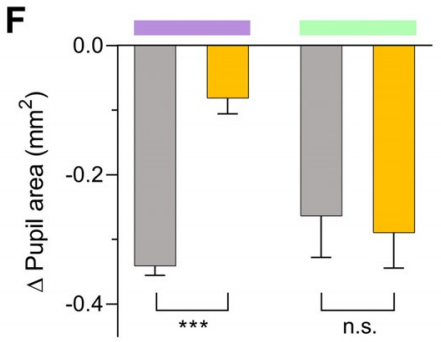

Figure 3. A,B) Pupillary responses in an isoflurane-anesthetized Opn $4 x R d 10$ blind mouse first treated with vehicle and then administered 1 mM adrenoswitch-1 in its right eye. Adrenoswitch-1 exerted mydriasis only upon UV illumination. Neither the vehicle nor UV light alone elicited any pupillary responses in the animals. A complete movie of the experiment (100x speed) is available as SI (Supplementary Movie S1). C) Change in area of vehicle vs. adrenoswitch-1 treated pupils of Opn $4 x R d 10$ blind mice at $t=20$ minutes from administration of the drug under UV

illumination. Data are means \pm SEM ( $n=4$ per condition). Statistical differences were determined by Student's $t$ test for unpaired observations. D) Pupillary responses in an isoflurane-anesthetized C57BL/6J wildtype mouse first treated with vehicle and then administered 1 mM adrenoswitch-1 in its right eye. The animal was exposed to 5-min cycles of UV, darkness and green light. UV and green light elicited a pupillary light reflex (PLR). Adrenoswitch-1 abolished the PLR only under concomitant UV illumination, while the response was maintained under visible light. E) Comparison of UV and green light evoked PLR in adrenoswitch-1-treated animals. Data are means \pm SEM ( $n=2$ per condition). F) PLR quantification in anesthetized wildtype mice. Pupil contraction evoked by exposure to UV and green light was compared between vehicle- and adrenoswitch-1-treated animals. Data are mean $\pm \operatorname{SEM}(n=2$ per condition). Statistical differences were determined by Student's $t$ test for unpaired observations.

and Supplementary Movie S1). These pupillary responses were reproducible in at least two cycles of alternating UV light and darkness, and were neither elicited by application of the vehicle nor by exposure to UV light alone. The maximum photoresponses were consistently observed after approximately $20 \mathrm{~min}$ from its administration (Figure 3B,C), in agreement with the pharmacodynamics of adrenergic ligands with similar structure and lipophilicity. ${ }^{[48]}$ As mydriasis is mediated by postsynaptic $\alpha_{1}$-adrenoceptors of the iris dilator muscle, the results of Figures $3 \mathrm{~A}-\mathrm{C}$ unambiguously demonstrate that adrenoswich-1 modulates endogenous ARs with light. Taking into account its molecular structure and the pharmacological characterization, we consider that the compound is likely to act as a partial agonist towards $\alpha_{1}$-ARs. Nonetheless, a thorough characterization of adrenoswitches pharmacological profiles would be needed to evaluate their specificity and selectivity over adrenergic and non-adrenergic receptors.
Finally, we tested if adrenoswitch-1 could be used in wildtype animals to selectively decouple pupil tone from enviromental illumination. As shown in Figures 3D-F, vehicle-treated C57BL/6J mice respond to violet and green light exposure with similar rapid PLRs. After topical administration of adrenoswitch-1, the miosis caused by UV light was blocked whereas the response to green light was maintained (Figures 3D-F). As the UV luminous stimulus is triggering a miotic reflex while simultaneously activating a photoregulated mydriatic agent (adrenoswitch-1), the two effects appear to cancel each other out. These pupillary responses were reproducible in all the cycles of alternating UV, darkness and green light and persisted after several washings of the cornea.

In contrast to anticholinergic mydriatics, the pharmacological action of adrenoswitch- 1 can be selectively controlled using specific wavelengths, can be fully reversed on demand, and does not alter the pupil basal tone. These unique 
properties might allow deeper insights into the role of PLR in vision adaptation dynamics. Moreover, as retinal responses are closely related to both area and intensity of the visual stimulus, adrenoswitches might become unique tools to investigate photoresponse kinetics and their mechanisms in retinal ganglion cells. ${ }^{[49]}$

On-demand regulation of pupil diameter might also have opthalmic applications. For exemple, slow-relaxing pre-illuminated adrenoswitches could induce a mydriatic response that spontaneously reverses upon progressive exposure of the eye to natural light. Procedures like examination of the retina or other structures of the eye fundus require fully dilated pupils. Although mydriasis can be readily achieved with topic cholinergic antagonists, post-exam pupil dilation lasts hours and causes photophobia, increased intra-ocular pressure and blurry vision. As these side effects are highly impairing, patients would benefit from drugs whose mydriatic effect can be rapidly reverted.

\section{Conclusion}

In summary, we have rationally designed novel azoheteroaryl photoswitches and characterised their adrenergic action in vitro and in vivo, including the control of locomotor activity in zebrafish larvae and pupil responses in mice. These results enable multiple applications that were previously unaccessible for adrenergic neurotransmission and open the way to achieve optimized photopharmacological properties based on this scaffold. On-demand modulation at specific locations might allow to single out adrenergic projections from the LC and to dissect their function in circuits and behaviour with high resolution. ${ }^{[50,51]}$ Moreover, adrenoswitches can enable photocontrol over a variety of physiological functions, like pupillary responses, and help unravel their physiology in vivo.

\section{Acknowledgements}

Mass spectrometry was performed at the IRB Barcelona Mass Spectrometry Core Facility, which actively participates in the BMBS European COST Action BM 1403 and is a member of Proteored, PRB2-ISCIII, supported by grant PRB2 (IPT13/0001-ISCIIISGEFI/FEDER). This research received funding from the European Union Research and Innovation Programme Horizon 2020 - Human Brain Project SG3 (945539), DEEPER (ICT-36-2020-101016787), Agency for Management of University and Research Grants/Generalitat de Catalunya (CERCA Programme; 2017-SGR-1442 and 2017-SGR-00465 projects), Fonds Européen de Développement Économique et Régional (FEDER) funds, Ministry of Science and Innovation (Grant PID2019-111493RBI00), Institute of Health Carlos III (IP18/00754, PI19/00443), Fundaluce and "la Caixa" foundations (ID 100010434, agreement LCF/PR/HR19/52160010) and Basque Government (IT-1211-19). The project Clúster Emergent del Cervell Humà (CECH, 001-P-001682) is co-financed by the European Union Regional Development Fund within the framework of the ERDF Operational Program of Catalonia 2014-2020 with a grant of $50 \%$ of total eligible cost. D.P. was supported by fellowship BES-2015-072657. A.M.J.G. was supported by fellowship BES-2017-083025.

\section{Conflict of interest}

The authors declare no conflict of interest.

Keywords: adrenergic receptors · azo compounds . neurotransmitters · photochromism · photopharmacology

[1] A. W. Tank, D. L. Wong, Compr. Physiol. 2015, 5, 1-15.

[2] E. Samuels, E. Szabadi, Curr. Neuropharmacol. 2008, 6, 254285.

[3] M. Llorca-Torralba, G. Borges, F. Neto, J. A. Mico, E. Berrocoso, Neuroscience 2016, 338, 93-113.

[4] S. J. Sara, Nat. Rev. Neurosci. 2009, 10, 211-223.

[5] J. M. Kim, J. Hwa, P. Garriga, P. J. Reeves, U. L. RajBhandary, H. G. Khorana, Biochemistry 2005, 44, 2284-2292.

[6] P. Makowka, T. Bruegmann, V. Dusend, D. Malan, T. Beiert, M. Hesse, B. K. Fleischmann, P. Sasse, Nat. Commun. 2019, 10, 1281.

[7] E. R. Siuda, J. G. McCall, R. Al-Hasani, G. Shin, S. Il Park, M. J. Schmidt, S. L. Anderson, W. J. Planer, J. A. Rogers, M. R. Bruchas, Nat. Commun. 2015, 6, 8480.

[8] S. Muralidharan, G. M. Maher, W. A. Boyle, J. M. Nerbonne, Proc. Natl. Acad. Sci. USA 1993, 90, 5199-5203.

[9] W. A. Velema, W. Szymanski, B. L. Feringa, J. Am. Chem. Soc. 2014, 136, 2178-2191.

[10] M. J. Fuchter, J. Med. Chem. 2020, 63, 11436-11447.

[11] K. Hüll, J. Morstein, D. Trauner, Chem. Rev. 2018, 118, 10710 10747.

[12] M. M. Lerch, M. J. Hansen, G. M. van Dam, W. Szymanski, B. L. Feringa, Angew. Chem. Int. Ed. 2016, 55, 10978-10999; Angew. Chem. 2016, 128, 11140-11163.

[13] P. Paoletti, G. C. R. Ellis-Davies, A. Mourot, Nat. Rev. Neurosci. 2019, 20, 514-532.

[14] R. K. Griffith in Foye's Principles of Medicinal Chemistry (Eds.: T. L. Lemke, D. A. Williams), Lippincott Williams \& Wilkins, Philadelphia, 2013, pp. 348-356.

[15] S. Pittolo, X. Gómez-Santacana, K. Eckelt, X. Rovira, J. Dalton, C. Goudet, J. P. Pin, A. Llobet, J. Giraldo, A. Llebaria, P. Gorostiza, Nat. Chem. Biol. 2014, 10, 813-815.

[16] M. Schoenberger, A. Damijonaitis, Z. Zhang, D. Nagel, D. Trauner, ACS Chem. Neurosci. 2014, 5, 514-518.

[17] F. Gentili, F. Ghelfi, M. Giannella, A. Piergentili, M. Pigini, W. Quaglia, C. Vesprini, P. A. Crassous, H. Paris, A. Carrieri, J. Med. Chem. 2004, 47, 6160-6173.

[18] G. Thomas, Medicinal Chemistry: An Introduction, Wiley, Hoboken, 2008, pp. 83-84.

[19] A. J. Nichols, R. R. Ruffolo, Alpha-Adrenoceptors: Molecular Biology, Biochemistry and Pharmacology, Karger, Basel, 1991, pp. $75-114$.

[20] D. Bléger, J. Schwarz, A. M. Brouwer, S. Hecht, J. Am. Chem. Soc. 2012, 134, 20597-20600.

[21] J. Calbo, A. R. Thawani, R. S. L. Gibson, A. J. P. White, M. J. Fuchter, Beilstein J. Org. Chem. 2019, 15, 2753-2764.

[22] J. Otsuki, K. Suwa, K. K. Sarker, C. Sinha, J. Phys. Chem. A 2007, 111, 1403-1409.

[23] S. Crespi, N. A. Simeth, B. König, Nat. Rev. Chem. 2019, 3, 133146.

[24] B. Civantos Calzada, A. A. De Artiñano, Pharmacol. Res. 2001, 44, 195-208.

[25] S. Guimarães, D. Moura, Pharmacol. Rev. 2001, 53, 319-356.

[26] P. M. Vanhoutte, J. Cardiovasc. Pharmacol. 2001, 38, 796-808. 
[27] L. Isaac, J. Cardiovasc. Pharmacol. 1980, 2, 5-20.

[28] E. G. Silva, T. Feres, L. M. Vianna, P. Okuyama, T. B. Paiva, J. Pharmacol. Exp. Ther. 1996, 277, 872-876.

[29] S. Iwanaga, O. Shibata, A. Tsuda, S. Hashimoto, T. Makita, S. Cho, K. Sumikawa, Res. Commun. Mol. Pathol. Pharmacol. 1998, 102, $137-147$

[30] E. F. Castillo, C. S. Ortíz, R. M. López, A. Ruíz, J. M. Vélez, C. Castillo, Fundam. Clin. Pharmacol. 2006, 20, 339-349.

[31] R. F. Furchgott, S. J. Ehrreich, E. Greenblatt, J. Gen. Physiol. 1961, 44, 499-519.

[32] K. L. Andrews, J. J. McGuire, C. R. Triggle, Br. J. Pharmacol. 2003, 138, $932-940$.

[33] F. W. Flitney, I. L. Megson, J. Physiol. 2003, 550, 819-828.

[34] J. A. Bauer, H. Fung, Pharmacol. Lett. 1993, 54, 1-4.

[35] J. C. Molin, L. M. Bendhack, Vascul. Pharmacol. 2004, 42, 1 -6.

[36] S. Regunathan, C. Youngson, H. Wang, D. J. Reis, Ann. N. Y. Acad. Sci. 1995, 763, 580-590.

[37] G. J. Molderings, M. Göthert, Gen. Pharmacol. 1999, 32, 17-22.

[38] M. F. Chen, J. T. Tsai, L. J. Chen, T. P. Wu, J. J. Yang, L. Te Yin, Y. L. Yang, T. A. Chiang, H. L. Lu, M. C. Wu, BioMed Res. Int. 2014, 2014, 1-7.

[39] D. Kokel, J. Bryan, C. Laggner, R. White, C. Y. J. Cheung, R. Mateus, D. Healey, S. Kim, A. A. Werdich, J. Stephen, C. A. Macrae, B. Shoichet, R. T. Peterson, Nat. Chem. Biol. 2010, 6, $231-237$.

[40] A. L. Rubinstein, Expert Opin. Drug Metab. Toxicol. 2006, 2, $231-240$.

[41] C. Matera, A. M. J. Gomila, N. Camarero, M. Libergoli, C. Soler, P. Gorostiza, J. Am. Chem. Soc. 2018, 140, 15764-15773.

[42] K. Rustler, G. Maleeva, A. M. J. Gomila, P. Gorostiza, P. Bregestovski, B. König, Chem. Eur. J. 2020, 26, 12722-12727.
[43] J. O. Ruuskanen, J. Laurila, H. Xhaard, V. V. Rantanen, K. Vuoriluoto, S. Wurster, A. Marjamäki, M. Vainio, M. S. Johnson, M. Scheinin, Br. J. Pharmacol. 2005, 144, 165-177.

[44] J. O. Ruuskanen, N. Peitsaro, J. V. M. Kaslin, P. Panula, M. Scheinin, J. Neurochem. 2005, 94, 1559-1569.

[45] J. Raczak-Gutknecht, T. Frackowiak, A. Nasal, R. Kaliszan, Pharmacol. Rep. 2013, 65, 305-312.

[46] Y. Yu, M. C. Koss, Auton. Neurosci. Basic Clin. 2005, 117, 17-24.

[47] H. Ishikawa, D. D. Miller, N. Patil, Naunyn-Schmiedeberg's Arch. Pharmacol. 1996, 354, $765-772$.

[48] H. C. A. Innemee, A. de Jonge, J. C. A. van Meel, P. B. M. W. M. Timmermans, P. A. van Zwieten, Naunyn-Schmiedeberg's Arch. Pharmacol. 1981, 316, 294-298.

[49] K. Y. Wong, F. A. Dunn, D. M. Berson, Neuron 2005, 48, 1001 1010.

[50] S. Pittolo, H. Lee, A. Lladó, S. Tosi, M. Bosch, L. Bardia, X. Gómez-Santacana, A. Llebaria, E. Soriano, J. Colombelli, K. E. Poskanzer, G. Perea, P. Gorostiza, Proc. Natl. Acad. Sci. USA 2019, 116, 13680-13689.

[51] G. Cabré, A. Garrido-Charles, M. Moreno, M. Bosch, M. Portade-la-Riva, M. Krieg, M. Gascón-Moya, N. Camarero, R. Gelabert, J. M. Lluch, F. Busqué, J. Hernando, P. Gorostiza, R. Alibés, Nat. Commun. 2019, 10, 907.

Manuscript received: August 1, 2020

Revised manuscript received: September 21, 2020

Accepted manuscript online: October 25, 2020

Version of record online: December 21, 2020 